\title{
Effects of Cocaine Self-Administration on Food-Reinforced Responding Using a Discrete Trial Procedure in Rats
}

\author{
WJ Lynch*,' and DCS Roberts' \\ 'Department of Physiology and Pharmacology, Wake Forest University School of Medicine, Winston-Salem, NC, USA
}

\begin{abstract}
Cocaine addiction has been characterized by a shift from controlled to uncontrolled and compulsive drug use. Using novel selfadministration procedures, we attempted to model this transitional phase and characterize the behavioral changes that underlie it. We chose to use food-reinforced responding across the light/dark cycle as an indicator of the degree to which cocaine was disrupting ongoing behavior as a potential measure of dysregulation. Four groups of rats $(n=5-6)$ were given $24-\mathrm{h}$ access to cocaine $(1.5 \mathrm{mg} / \mathrm{kg} / \mathrm{inj})$ available in 2, 3, 4, or 5 discrete trials/h. All rats were given continuous access to a second lever that resulted in the delivery of a $45 \mathrm{mg}$ food pellet under a fixed ratio I schedule. The results showed that under low access conditions (eg 2 discrete trials/h), both food- and cocainereinforced responding were diurnally regulated and occurred coincidentally. As access to cocaine was increased, there was a progressive disruption in the diurnal control over both food- and cocaine-maintained responding. High access conditions also produced transient decreases in the total levels of food-reinforced responding. These findings suggest that high access to cocaine under the discrete trial cocaine self-administration procedure produces a transient disruption in the diurnal control over behavior maintained by food and that the level of control (or loss of) may be a useful marker of dysregulation.

Neuropsychopharmacology (2004) 29, 669-675, advance online publication, 17 December 2003; doi: I 0. I 038/sj.npp. I 300363
\end{abstract}

Keywords: addiction; animal model; cocaine; discrete trial; self-administration; food

\section{INTRODUCTION}

Cocaine addiction has been characterized by a transition from controlled, recreational use to uncontrolled and compulsive patterns of use (Gawin and Kleber, 1985). There has been a recent emphasis on developing animal cocaine self-administration procedures that model this transitional process from use/abuse to addiction. Several new models have been developed (eg Ahmed and Koob, 1998, 1999; Tornatzky and Miczek, 2000; Roberts et al, 2002), and it appears that the frequency of access to cocaine is a critical factor in producing a transition from controlled, regulated intake, to uncontrolled and dysregulated patterns of intake. For example, under the short access conditions that are typically used in animal drug self-administration studies (ie 1-3 h sessions), levels of daily cocaine intake are stable, and they remain so over months of testing (Yokel, 1987). In contrast, recent research has shown that when access conditions are increased above those used by traditional methods, changes in self-administration behaviors are observed. For example, Ahmed and Koob (1998)

\footnotetext{
*Correspondence: WJ Lynch, Department of Psychiatry, Yale University School of Medicine, 34 Park St., New Haven, CT 06508, USA, E-mail: wendy.lynch@yale.edu

Received 16 April 2003; revised 16 October 2003; accepted 29 October 2003

Online publication: 30 October 2003 at http://www.acnp.org/citations/ Npp 10300303 | 65/default.pdf
}

have shown that increasing the session length from 1 to $6 \mathrm{~h}$ can produce escalating patterns of intake. Similarly, Tornatzky and Miczek (2000) have examined the effect of a very long session $(72 \mathrm{~h})$ on patterns of intake and they have reported a progressive deterioration in the mechanisms that regulate intake over time. During the first $8-10 \mathrm{~h}$, responding is characterized by uniformly spaced infusions but beginning at hour 22-24, responding becomes erratic. Owing to toxicity, these studies must be truncated to only a few days of continuous access.

This laboratory has used a discrete trial procedure, which allows access to cocaine throughout the light/dark cycle, to study the effects of high cocaine intake (Fitch and Roberts, 1993; Morgan et al, 2002; Roberts et al, 2002). With this procedure, rats have 24-h access 1 day to cocaine injections that are available in discrete 10-min trials. The number of such trials per hour and the unit injection dose can be manipulated to produce a variety of patterns of intake. Under low access conditions (ie 2 trials $/ \mathrm{h}, 1.5 \mathrm{mg} / \mathrm{kg} / \mathrm{inj}$ ), the daily intake is stable and responding occurs predominantly during the dark phase of the light/dark cycle. Under high access conditions ( $4-5$ trials $/ \mathrm{h}, 1.5 \mathrm{mg} / \mathrm{kg} / \mathrm{inj})$, selfadministration behavior becomes desynchronized from the light/dark cycle and intake is characterized by a 'bingeabstinent' pattern of use, with animals taking virtually every infusion available for the first 1-2 days, followed by periods of self-imposed drug abstinence that are interspersed with periods of active drug use. An extensive parametric analysis 
has revealed that as access to cocaine increases, the levels of intake increase and become dysregulated and the diurnal control over self-administration behavior decreases (Roberts et al, 2002). These findings may reflect features of cocaine addiction, namely a loss of control over use and dysregulated patterns of use.

In this study, we were interested in exploring the effects of such access conditions on changes in the diurnal control and regulation (eg temporal patterns across light/dark cycle) of other behaviors. We chose to use food-reinforced responding as an indicator of the degree to which cocaine was disrupting ongoing behavior in the hope that it would provide an ethologically relevant measure of dysregulation. We have previously shown that when access to cocaine is limited to 2 trials/h, both cocaine and food-reinforced responding are diurnal and coexist during the dark phase of the light/dark cycle (Roberts and Andrews, 1997). However, the temporal patterns of food-reinforced responding as a function of cocaine self-administration are not yet known. Thus, the purpose of the present experiment was to investigate the effect of cocaine self-administration under various discrete trial conditions $(2,3,4$, and 5 trials/h) on food-reinforced behavior.

\section{METHODS}

\section{Subjects}

Male Sprague-Dawley rats (Harlan, IN, USA) weighing between 300 and $350 \mathrm{~g}$ at the start of the experiment were pair-housed in hanging stainless-steel home cages with free access to food and water. Rats were allowed a minimum of 3 days to acclimate to the facility before any procedures or testing began. Subsequently, rats were individually housed in $25 \times 25 \times 25 \mathrm{~cm}^{3}$ testing chambers where they remained for the duration of the experiment. Food ( $45 \mathrm{mg}$ Noyes nutritionally balanced pellets) was continuously available under a fixed ratio 1 schedule, except during cocaine selfadministration training when Purina Laboratory Chow was available ad libitum. Animals were maintained on a reverse light/dark cycle (lights on at 1500) and had free access to water throughout all phases of the experiments. All research was approved by the Animal Care and Use Committee at Wake Forest University and was conducted in accordance with the guidelines set by the National Institutes of Health.

\section{Experimental Procedures}

Food self-administration training. Rats had continuous access to $45 \mathrm{mg}$ Noyes pellets under a fixed ratio 1 schedule. All animals began to operate the food-associated lever (left lever) within $24 \mathrm{~h}$ without explicit training. The food cup and feeding apparatus were inspected daily to ensure that the pellets were being delivered properly. The basal level of food-maintained responding was measured for an initial period of 7 days. Subsequently, each rat was prepared with a chronically indwelling catheter using methods that have been described previously (Lynch et al, 2001). Following surgery, each animal was placed back in its operant chamber, the food-associated lever was retracted, and standard food pellets were available ad libitum.
Cocaine self-administration training. Cocaine self-administration training began the day after surgery at 1000 . At the beginning of each daily training session, the cocaineassociated lever (right lever) was extended into the operant chamber and responding was reinforced under a fixed ratio 1 schedule. Each rat received one 'priming' infusion at the beginning of each training session. At the start of each infusion, the lever was retracted and a stimulus light located above the cocaine-associated lever was illuminated for the duration of the infusion (approximately $5 \mathrm{~s}$ ) and for an additional $20 \mathrm{~s}$ to signal a time-out period. After the $20 \mathrm{~s}$ time-out period, the lever was extended into the chamber. Cocaine infusions $(1.5 \mathrm{mg} / \mathrm{kg})$ were available until a total of 40 infusions were self-administered. Responding for cocaine under the fixed ratio schedule was assessed daily until rats met the criteria for acquisition. The acquisition of cocaine self-administration was defined as three consecutive sessions in which rats earned all 40 infusions that were available.

Concurrent food and cocaine self-administration. Once rats acquired cocaine self-administration under the fixed ratio schedule, the food-associated lever was reintroduced and concurrent responding for cocaine (fixed ratio 1, 40 injections max, $1.5 \mathrm{mg} / \mathrm{kg} /$ infusion) and food (fixed ratio 1 , unlimited access) was assessed for two consecutive sessions. Subsequently, rats were randomly assigned to one of four cocaine access conditions in which they received $2,3,4$, or 5 discrete trials/h with concurrent access to food (fixed ratio 1, unlimited access). Several animals were also tested under a 6 discrete trials/h condition; however, since toxicity (eg rapid weight loss, cessation of feeding, and grooming behaviors) became evident quickly under this condition, it was not included in the final experimental design. Each 10min discrete trial was initiated by the introduction of the cocaine-associated (right) lever into the chamber. Each response on the right lever during a 10-minute trial produced an infusion of cocaine $(1.5 \mathrm{mg} / \mathrm{kg} /$ infusion $)$, and the stimulus light above the lever was illuminated for the $20 \mathrm{~s})$. A discrete trial was terminated and the lever was retracted following a response or after $10 \mathrm{~min}$ had elapsed. Trials were initiated at regular intervals beginning every 30 , 20,15 , or $12 \mathrm{~min}$ for the discrete trial $2,3,4$, and $5 \mathrm{trial} / \mathrm{h}$ access conditions, respectively. Discrete trial sessions continued for a total of 14 consecutive days. In a $24-\mathrm{h}$ period, the total number of trials and corresponding total number of cocaine infusions available were $48,72,96$, and 120 for the discrete trials $2,3,4$, and 5 access conditions, respectively. 'Priming' infusions were never given on any discrete trial session. The food-associated lever was extended into the chamber for the duration of the discrete-trial sessions, and each response on it resulted in the delivery of a single food pellet. Animals that lost cannula patency were replaced to achieve a final $N$ of five to six per group.

\section{Data Analysis}

The effects of cocaine on food-reinforced behavior were examined across various discrete trial cocaine access conditions. The dependent measures were total hourly and daily levels of responding for food and cocaine and the 
percent of total daily levels of responding that occurred in the dark phase for both food and cocaine. Repeated measures ANOVA was used to compare hourly levels of food- and cocaine-reinforced responding across the different discrete trials conditions averaged across the initial 7 days and the last 7 days under the discrete trial procedure. The percent of total daily levels of responding for food and cocaine that occurred within the dark phase was determined for each subject and each day, and group means were compared using repeated measures ANOVA. Separate paired $t$-tests were used to compare the average total levels of food-reinforced responding and percent of total responding that occurred within the dark phase between the baseline and concurrent food and discrete trial cocaine sessions within each group. A record of cocaine-reinforced and nonreinforced trials was obtained for each trial and for each animal, and these records were compared to the corresponding records of temporal responding for food in order to determine whether food- and cocaine-reinforced responding occurred within the same time frame. All statistical analyses were conducted using SPSS 10, and findings were considered statistically significant if $p$-values were less than 0.05 .

\section{RESULTS}

Under baseline conditions, food-reinforced responding was diurnally regulated and it was characterized by high levels of responding during the dark phase that decreased to low levels at the onset of the light phase and then slowly increased to peak levels at the onset of the dark phase (data not shown). Under the five baseline food-reinforced sessions, an average of $72 \pm 2 \%$ of the total amount of responding occurred within the dark phase, and the average daily number of pellets obtained was $584 \pm 15$.

Figure 1 illustrates the effect of cocaine self-administration under the discrete trial procedure on the diurnal control of food- and cocaine-reinforced responding during the first and the second week of access. Although the groups did not differ on patterns or levels of responding for food under baseline (food only) conditions prior to cocaine selfadministration $(p>0.05)$, the differences were initially observed under the discrete trial procedure. Specifically, during the first week of access to cocaine, there was a dosedependent disruption in diurnal control over food-maintained responding (eg hourly distribution of responding across light/dark cycle) and a dose-dependent decrease in the daily number of pellets (see Table 1). However, these effects were diminished or not apparent during the second week of testing. Specifically, a repeated measures ANOVA comparing the average hourly number of pellets at baseline with those obtained during days 1-7 and days 8-14 under the discrete trial cocaine self-administration procedure revealed a significant main effect of Condition (eg Baseline, Days 1-7, and Days 8-14; $(\mathrm{F}(2,34)=9.9, p<0.001))$ and Hour $(\mathrm{F}(23,391)=20.3, p<0.001)$, and a significant interaction of Condition by Group $(\mathrm{F}(6,34)=2.6, p<0.05)$, Hour by Group $(\mathrm{F}(69,391)=2.5, p<0.001)$, Condition by Hour $(\mathrm{F}(46,782)=4.7, p<0.001)$, and a trend for a main effect of Group $(p=0.06)$ and the interaction of Condition by Hour by Group $(p=0.07)$. Subsequent analyses comparing the baseline hourly number of food pellets to those obtained on days 1-7 within each discrete trial access group revealed a significant effect of Condition for the 3 discrete trial/h group $(\mathrm{F}(1,4)=10.8, p<0.05), 4$ discrete trial/h group $(\mathrm{F}(1,4)=14.3, p<0.05)$, and 5 discrete trial/h group $(\mathrm{F}(1,5)=72.8, p<0.001)$, but not for the 2 discrete trial/h group $(p>0.05)$. However, similar analyses comparing patterns of food pellets at baseline to those obtained on days 8-14 revealed a nonsignificant effect of Condition for all discrete trial cocaine self-administration groups $(p>0.05)$.

The patterns and levels of cocaine-reinforced responding also changed over time. Increased access produced a proportionally greater daily intake of cocaine (see Table 1) and a dose-dependent disruption in the diurnal control over cocaine-reinforced responding. These effects were most pronounced during the first week of exposure. Repeated measures ANOVA comparing hourly cocaine intake averaged across days 1-7 with hourly cocaine intake averaged across days 8-14 within each group revealed a significant effect of Week within the 4 discrete trial/h group $(\mathrm{F}(1,4)=14.4, \quad p<0.05)$, and 5 discrete trial/h group $(\mathrm{F}(1,5)=12.4, p<0.05)$, but not the 2 or 3 discrete trial/h groups $(p>0.05)$. A comparison of the records of cocainereinforced responding with records of food-reinforced

Table I Summary of the Daily Mean ( \pm SEM) Number of Food Pellets and the Mean $( \pm$ SEM) Percent of Total Responding that Occurred for Food Averaged Across the Five Baseline Sessions (Food Only) and Across the First and the Last Seven Sessions Under the Discrete Trial Cocaine Self-Administration Procedure as a Function of Cocaine Access Group

\begin{tabular}{|c|c|c|c|c|c|c|c|c|}
\hline \multirow[b]{2}{*}{ Group } & \multicolumn{3}{|c|}{ Number of food pellets } & \multicolumn{3}{|c|}{ Food in dark (\%) } & \multicolumn{2}{|c|}{ Number of infusions } \\
\hline & Baseline & Days I-7 & Days 8-14 & Baseline & Days I-7 & Days 8-14 & Days I-7 & Days 8-14 \\
\hline 3 discrete trials/h & $595 \pm 36$ & $462 \pm 28 *$ & $503 \pm 81$ & $73 \pm 3$ & $61 \pm 3 *$ & $66 \pm 7$ & $32 \pm 4$ & $34 \pm 2$ \\
\hline 4 discrete trials/h & $609 \pm 15$ & $502 \pm 44 *$ & $574 \pm 47$ & $73 \pm 4$ & $57 \pm 4$ *** & $68 \pm 2$ & $65 \pm 1$ & $57 \pm 3 * * * * *$ \\
\hline
\end{tabular}

The mean $( \pm$ SEM) number of cocaine infusions averaged across the first and the last seven sessions under the discrete trial cocaine self-administration for each group is also presented.

*Compared to baseline levels $(p<0.05)$. *** Compared to baseline levels $(p=0.06)$. *** Compared to days $1-7$ ( $p<0.05)$. 

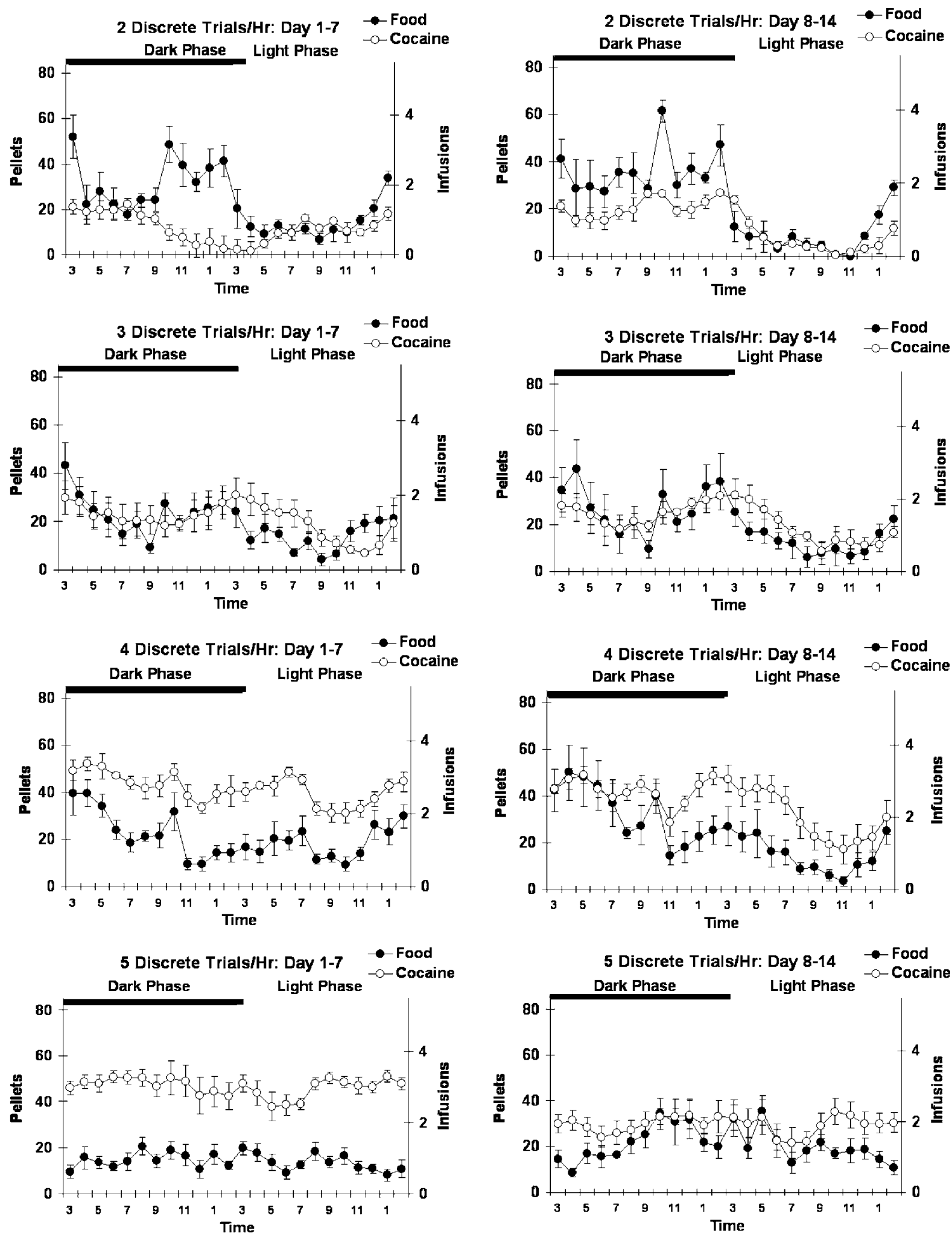

Figure I Effect of cocaine access on changes in the diurnal control over food and cocaine self-administration behavior over the first 7 and last 7 days of discrete trial exposure. Mean ( + SEM) hourly number of food pellets (filled symbols) and cocaine infusions (open symbols) obtained across the light/dark cycle (lights on at I 500) averaged across days I-7 (left panels) and days 8-14 (right panels) of the discrete trial self-administration procedure for each of the four cocaine access conditions. The horizontal lines mark the hours within the dark phase. Each data point represents the mean of 5 (2, 3, and 4 discrete trials/h groups) and six (5 discrete trials/h group) rats. 

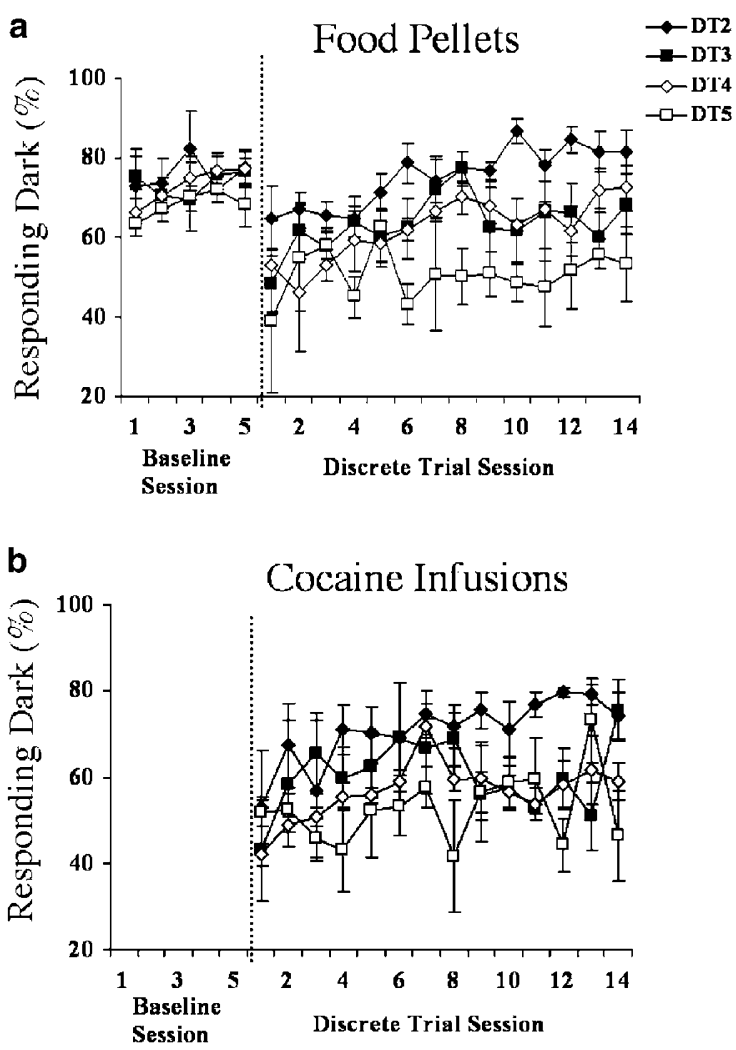

Figure 2 Effect of discrete trial cocaine self-administration on diurnal control over days. (a) Mean ( \pm SEM) percent of total food-reinforced responding that occurred in the dark phase during the five baseline testing sessions (food alone) and during the 14 discrete trial testing sessions for each cocaine access group. (b) Mean $( \pm S E M)$ percent of total cocaine infusions obtained in the dark phase during the 14 discrete trial testing sessions for each cocaine access group. Each data point represents the mean of 5 (2, 3, and 4 discrete trials/h groups) and six (5 discrete trials/h group) rats.

responding revealed that even under high access conditions (5 discrete trials $/ \mathrm{h}$ ), approximately $85 \%$ of all foodreinforced responding occurred within the same time frame as cocaine-reinforced responding (ie during the 10-min trial plus the 2-min time-out), suggesting that responding for food was not occurring during cocaine abstinence periods. Thus, as access to cocaine increased, initially the diurnal control over both cocaine- and food-maintained responding was disrupted, the number of pellets obtained was decreased, and the total number of cocaine infusions was increased. Additionally, food-reinforced responding occurred coincidentally with cocaine-reinforced responding.

Figure 2 further illustrates the diurnal nature of responding for food and cocaine as a function of group. Under baseline conditions, approximately $72 \%$ of foodreinforced responding occurred during the dark phase of the light-dark cycle. Under the discrete trial cocaine selfadministration procedure, there was a dose-dependent reduction in the diurnal control over food self-administration behavior (see Figure 2a). However, the level of disruption in the diurnal control over responding decreased over days across all cocaine access conditions. That is, the percent of total responding that occurred within the dark phase progressively increased over time. A repeated measures ANOVA comparing the percent of total respond- ing that occurred in the dark phase over the 14 days as a function of group revealed a significant main effect of Group $(\mathrm{F}(3,17)=10.35, p<0.05)$, and Day $(\mathrm{F}(13,221)=1.95$, $p<0.05)$, but a nonsignificant interaction of Group by Day $(p>0.05)$. Compared to baseline, the percent of total responding for food that occurred in the dark phase during the first 7 days under the discrete trial procedure was significantly decreased under the $3(t=4.0, \mathrm{df}=4, p<0.05)$ and $5(t=2.8, \mathrm{df}=5, p<0.05)$ discrete trial/hour condition, with a trend for a decrease under the 4 discrete trial $/ \mathrm{h}$ condition $(t=2.7, \mathrm{df}=4, p=0.06)$, but was not significant under the 2 discrete trial/hour condition $(p>0.05)$. However, during the last 7 days under the discrete trial procedure, compared to baseline, the percent of total responding for food that occurred in the dark phase was significantly decreased only under the highest access conditions $(t=4.6, \mathrm{df}=5, p<0.01)$ (see Table 1). Thus, during the last 7 days of access under the discrete trial procedure, most animals had regained diurnal control over food-reinforced responding (ie responding was becoming more selective to the dark phase and was similar to the diurnal patterns observed under baseline conditions). However, under the highest access conditions (eg 5 discrete trials/h), food-reinforced responding remained disrupted and it occurred equally within the light and dark phases.

Similarly, there was a dose-dependent disruption in the diurnal control of cocaine self-administration behavior (see Figure 2b). As with food-reinforced responding, there was a progressive increase in the percent of the total cocainereinforced responding that occurred within the dark phase. A repeated measures ANOVA comparing the percent of total responding that occurred in the dark phase over the 14 days as a function of group revealed a significant main effect of Group $(\mathrm{F}(3,17)=8.27, p<0.01)$ and Day $(\mathrm{F}(13,221)=$ $1.83, p<0.05)$, but a nonsignificant interaction of Group by Day $(p>0.05)$. Thus, the diurnal control over both foodand cocaine-maintained behavior became progressively greater over time, but food-maintained responding continued to show some signs of dysregulation across the entire testing period under the highest access conditions.

In order to assess the effect of cocaine intake on the health of the animals, several rats from each of the 2, 3, 4, and 5 discrete trial/h groups ( $n=2,5,3$, and 4 , respectively) were weighed at various time points throughout the study (eg before and during the initial seven and last seven discrete trial sessions). Although animals did not differ in weight prior to cocaine self-administration under the discrete trial procedure, animals in the high access conditions showed a transient decrease in body weight during the initial discrete trial sessions that returned to baseline levels during the later sessions. A repeated measures ANOVA revealed a nonsignificant main effect of Group $(p>0.05)$ and Time $(p>0.05)$, but a significant Group by Time interaction $(\mathrm{F}(6,34)=2.68, p<0.05)$. An ANOVA comparing body weights during the later sessions revealed no significant differences between groups $(p<0.05)$.

\section{DISCUSSION}

The purpose of the present experiment was to investigate whether food-reinforced responding is an indictor of 
dysregulation in animals with concurrent access to cocaine. The results revealed that under conditions that promote stable levels of cocaine intake, behavior maintained by food was also stable and diurnally regulated. However, under conditions that promote uncontrolled and dysregulated patterns of cocaine intake, the behavior maintained by food was dysregulated from the normal diurnal pattern. High access conditions also produced decreases in the total levels of food-reinforced responding. These effects became progressively greater with increases in cocaine access and were more robust during the initial days of exposure to cocaine. These findings suggest that under high access conditions, the diurnal control over behavior maintained by food decreases and that the level of control (or loss of) may be a useful marker of dysregulation. However, the decrease in diurnal control over food-maintained behavior was only persistent under the highest access conditions and even there it showed some signs of recovery.

The finding of a dose-dependent decrease in the diurnal control over food-reinforced responding is consistent with our recent characterization of patterns of cocaine selfadministration under the discrete trial procedure (Roberts et al, 2002). We found evidence of dysregulation when access to cocaine exceeded 2 discrete trials/h. Under these conditions, the initial patterns of responding for food and cocaine were desynchronized from the 'normal' diurnal pattern and responding was equally likely to occur within the light and dark phases. As access to cocaine increased, the initial period of dysregulation became longer, and under the highest access condition ( 5 discrete trials/h), evidence of dysregulation was apparent for behavior maintained by food throughout the entire 14 days of testing, with responding occurring equally within the light and dark phases.

Interestingly, the effects of cocaine on patterns of foodmaintained responding were transient and the time frame of dysregulation corresponded to the period of time that cocaine-reinforced behavior was dysregulated. We have previously shown that the 'normalization' of cocaine selfadministration behavior that occurs under the discrete trial procedure over time may be due to the development of tolerance to the reinforcing effects of cocaine. Specifically, following cocaine self-administration under high access conditions, levels of responding under the progressive ratio schedule are decreased and rates of responding under the fixed ratio schedule are increased (Roberts et al, 2002; Morgan et al, 2002). These findings are consistent with an interpretation of a reduced drug effect. In the current study, the 'normalization' of food intake may be due to the decreases in cocaine intake over time. Specifically, the greatest levels of cocaine intake were observed during the initial 1-3 days under the discrete trial procedure. It is during this initial period that the greatest levels of dysregulation were observed for food-maintained responding. After this initial period of high drug intake, the average daily cocaine intake decreased and stabilized at a lower level and food-maintained responding increased and became more diurnally regulated. Notably, under the highest access condition, although daily levels of cocaine intake decreased, both food- and cocaine-maintained responding continued to be dysregulated.

It is also possible that tolerance developed to the disruptive effects of cocaine on food-maintained respond- ing. Evidence from humans and animals show that tolerance develops rapidly to the anorectic effects of stimulant drugs (Foltin and Fischman, 1991; Kuo and Cheng, 2002). Here, we found that levels of food-maintained responding were markedly decreased during the initial exposure under the discrete trial procedure, but that beyond the first 1-2 days, cocaine self-administration failed to suppress food intake. The present results showed that neither food intake nor body weights differed between groups during the later discrete trial sessions, even though each group varied considerably in terms of total cocaine intake. It seems that tolerance developed to the food-suppressant effects of cocaine by the second or third day under the 5 discrete trial/h condition. This finding is consistent with our previous demonstration that tolerance develops to the reinforcing effects of cocaine after only a few days under a 5 discrete trial/h schedule (Roberts et al, 2002). Thus, while tolerance develops quickly to the food suppressant effect of cocaine, the disruptive effects on the diurnal cycle continue. Taken together, these results suggest that behavior may become disorganized (dysregulated) long before something so critical for survival as feeding becomes suppressed. By the time feeding is suppressed, cocaine selfadministration is probably at a toxic stage.

Human cocaine users have been reported to exhibit 'binges/abstinent cycles', wherein cocaine is taken in bouts that last for many hours or days (Gawin and Kleber, 1985; Gawin, 1989, 1991). Abstinence periods between successive binges vary in length and have been characterized as a dysphoric mood state accompanied by changes such as hypersomnia, insomnia, and increased appetite (Diagnostic and Statistical Manual of Mental Disorders (DSM-IV); Gawin and Kleber, 1985; American Psychiatric Association, 1994; Gawin, 1989, 1991; Weddington et al, 1990; Johanson et al, 1999). Based on this literature, we expected that foodmaintained behavior would occur predominantly during periods of low drug intake and would predict a recuperative, drug-abstinent period. Contrary to this prediction, after the initial 'binge' in which animals took virtually every infusion available for the first 1-3 days, food-reinforced responding co-occurred with cocaine self-administration rather than during a recuperative phase-with approximately $85 \%$ of food-reinforced responding occurring within the same time frame as cocaine-reinforced responding. We knew that food intake could co-occur with cocaine selfadministration under a 2 discrete trial/h condition (Roberts and Andrews, 1997). However, given the extreme levels of daily cocaine intake $(88 \mathrm{mg} / \mathrm{kg} /$ day $)$ under a 5 discrete trial $/ \mathrm{h}$ condition, we expected that food intake would be suppressed. It should be noted, however, that food-reinforced responding is not a direct measure of eating. Although food cups were inspected at least once daily and were found to contain no more than a few pellets, and were generally empty, it is still possible that animals were collecting pellets and eating them at a later time (perhaps during an abstinence period). Future research is necessary to explore this issue fully.

In summary, we have previously shown that increasing the number of trials/h produces a dose-dependent disruption of diurnal patterns of cocaine intake. The present results extend these findings to include a dose-dependent disruption in the diurnal pattern of food-maintained 
behavior. High access under the discrete trial procedure results in high levels of drug intake and a loss of diurnal control over both food- and cocaine-maintained behavior. These findings suggest that the level of diurnal control (or loss of) may be a useful maker of dysregulation. Notably, we have previously shown that access to cocaine under the 4 discrete trial/h condition produces marked increases in motivation to obtain cocaine, but only after an extended forced abstinence period (Morgan et al, 2002). Taken together, the findings suggest that high intake as was observed here, coupled with a forced abstinence period, may capture the compulsive features that are characteristic of human cocaine addiction.

\section{ACKNOWLEDGEMENTS}

We are grateful to April Engram for her technical assistance and to Dr Theresa Lee for her critical comments on a previous version of this manuscript. We would like to acknowledge the Yale Interdisciplinary Women's Health Research Scholar Program on Women and Drug Abuse, NIH Office of Research on Women's Health. This work was supported by NIDA Grants RO1DA14030 and P50DA06634 (DCSR) and T32DA07246 (WJL).

\section{REFERENCES}

Ahmed SH, Koob GF (1998). Transition from moderate to excessive drug intake: change in hedonic set point. Science 282: 298-300.

Ahmed SH, Koob GF (1999). Long-lasting increase in the set point for cocaine self-administration after escalation in rats. Psychopharmacology 146: 303-312.

American Psychiatric Association (1994). Diagnostic and Statistical Manual of Mental Disorders, 4th edn. American Psychiatric Publishing, Inc.: Washington, DC.

Fitch TE, Roberts DCS (1993). The effects of dose and access restrictions on the periodicity of cocaine self-administration in the rat. Drug Alcohol Depend 33: 119-128.
Foltin RW, Fischman MW (1991). Methods for the assessment of abuse liability of psychomotor stimulants and anorectic agents in humans. Br J Addict 86: 1633-1640.

Gawin FH (1989). Cocaine abuse and addiction. J Fam Pract 29: 193-197.

Gawin FH (1991). Cocaine addiction: psychology and neurophysiology. Science 251: 1580-1586.

Gawin FH, Kleber HD (1985). Cocaine abuse in a treatment population: patterns and diagnostic considerations. In: Kozel NJ, Adams EH (eds). Cocaine Use in America: Epidemiologic and Clinical Perspectives, NIDA Res Monogr 61. US Government Printing Office: Washington, DC. pp 182-193.

Johanson CE, Roehrs T, Schuh K, Warbasse L (1999). The effects of cocaine on mood and sleep in cocaine-dependent males. Exp Clin Psychopharmacol 7: 338-346.

Kuo DY, Cheng JT (2002). Role of cerebral dopamine but not plasma insulin, leptin and glucocorticoid in the development of tolerance to the anorectic effect of amphetamine. Neurosci Res 44: 63-69.

Lynch WJ, Roth ME, Mickelberg JL, Carroll ME (2001). Role of estrogen in the acquisition of intravenously self-administered cocaine in female rats. Pharmacol Biochem Behav 68: 641-646.

Morgan D, Brebner K, Lynch WJ, Roberts DCS (2002). Increases in the reinforcing efficacy of cocaine after particular histories of reinforcement. Behav Pharmacol 13: 389-396.

Roberts DCS, Andrews MM (1997). Baclofen suppression of cocaine self-administration: demonstration using a discrete trials procedure. Psychopharmacology 131: 271-277.

Roberts DCS, Brebner K, Vincler M, Lynch WJ (2002). Patterns of cocaine self-administration in rats produced by various access conditions under a discrete trials procedure. Drug Alcohol Depend 67: 291-299.

Tornatzky W, Miczek KA (2000). Cocaine self-administration 'binges': transition from behavioral and autonomic regulation toward homeostatic dysregulation in rats. Psychopharmacology 148: 289-298.

Weddington WW, Brown BS, Haertzen CA, Cone EJ, Dax EM, Herning RI et al (1990). Changes in mood, craving, and sleep during short-term abstinence reported by male cocaine addicts. A controlled, residential study. Arch Gen Psychiatry 47: 861-868.

Yokel RA (1987). Intravenous self-administration: response rates, the effects of pharmacological challenges, and drug preference. In Bozarth MA (ed). Methods of Assessing the Reinforcing Properties of Abused Drugs. Springer-Verlag: New York. pp 1-33. 